\title{
An efficient image denoising method based on KPDE
}

\author{
Abhishek Dipak Shroff ${ }^{1^{*}}$, Kailash Patidar ${ }^{2}$ and Harsh Pratap Singh ${ }^{3}$ \\ M.Tech Scholar, Department of Computer Science, School of Engineering, Sri Satya Sai University of Technology \\ $\&$ Medical Sciences, Sehore, Madhya Pradesh, India ${ }^{1}$ \\ Professor and HOD, Department of Computer Science, School of Engineering, Sri Satya Sai University of \\ Technology \& Medical Sciences, Sehore, Madhya Pradesh, India ${ }^{2}$ \\ Assistant Professor, Department of Computer Science, School of Engineering, Sri Satya Sai University of \\ Technology \& Medical Sciences, Sehore, Madhya Pradesh, India ${ }^{3}$
}

\section{(C2018 ACCENTS}

\begin{abstract}
In this paper a $k$-means based PDE has been applied for image denoising. In this approach first data pre-processing mechanism has been applied. The next procedure is for the image denoising. In this process the pre-processed image has been selected. Gaussian noise has been added in terms of noise percentage. Then object based clustering and decomposition has been applied for efficient data point selection. For this $k$-means algorithm has been applied. By this process object point cluster has been obtain. The main benefit by this approach is it is able in finding the decomposition as well as the similar point by the similarity ranking and matching. PDE-FFT hybridization has then been applied on the clustered data for the final noise separation. Then the PSNR values have been calculated for the comparative study. The results indicated that our approach has the capability in better noise removal in terms of previous method.
\end{abstract}

\section{Keywords}

Image denoising, K-means, PDE-FFT, PSNR.

\section{Introduction}

Image denoising methods are helpful in improving the efficiency in removing the noise from input image dataset. Correspondence of computerized pictures through the remote sensors or on the other hand organizes turned into a costly errand, because of the misfortune of data in the correspondence way that outcomes in poor remaking. By and large, remaking of a flag is finished by Shannon examining hypothesis. It expresses that reproduction is conceivable if and just if the flag is transmitted with Nyquist rate [1-3].

On account of pictures, the number of tests is high. Transmission of those numerous examples with twofold information rate is a mind boggling errand and elucidation of tests requires exceptionally designed machines [4]. All these factors make computerized picture correspondence as unpredictable and costly correspondence frameworks [5].

*Author for correspondence
According to Nyquist/Shannon inspecting hypothesis, the inspecting rate ought to be no less than double the most extreme recurrence exhibit in the flag [6, 7]. Compacted detecting permits as to test the information well underneath the Nyquist rate. Truth be told, compacted detecting hypothesis empowers a 4-overlap lessening in obtaining time for biomedical pictures by permitting the undersampling [8]. It has numerous applications in various fields, including medical imaging, seismic imaging, dispersed and remote detecting, analog to digital conversion [9-11].

Along these lines, it can successfully decorrelate the conditions found over picture discontinuities and minimal high recurrence parts incited by picture highlights into the lower level or low band pass.

In the event that we consider the wavelet change then it can be adequately catch particular indicates up two measurements implies including one measurement, yet it is flop in speaking to the real highlights like edge , shading ,shape et cetera. There are a few directional and non-directional repetitive changes which are investigated in various research papers, 
including the curvelet, contourlet, wedgelet, bandlet, and the steerable wavelet [12-14].

There are a few methodologies which are fundamentally worry with denoise a picture information, for example, averaging channel, Median channel, Gaussian channel and partial differential equations (PDE) approach. On the off chance that we investigate the properties of good pictures then it will be with the fewer noise and limit the obscure or obscure lessening is the imperative factor. The PDE approach is much viable and applies in a few researches like $[15,16]$. Be that as it may, it is more powerful on the off chance that we apply fourth request fractional differential condition. Utilizations of the PDE models can be generally found in a wide scope of picture reclamation errands, for example, denoising and improvement [17] shading picture handling and determination. This gives us the future knowledge or work with the forward request fractional differential condition with a similar request toward obscure decrease.

In this paper an efficient denoising approach has been presented.

\section{Literature review}

In 2011, Lang et al. [18] proposed a joined transform picture denoising calculation in view of morphological component analysis (MCA). They have suggested that the approach is helpful in the image separation into natural scene and linear singular structure. Curvelet change limit denosing is utilized as a part of straight particular structure while wavelet change manages smooth part. This calculation makes full utilization of individual points of interest of the wavelet change and curvelet change. Investigation comes about demonstrate that the calculation can better keep up the points of interest qualities in managing the picture with straight peculiarity, and it has a superior denosing execution for picture than a basic wavelet thresholding or curvelet thresholding.

In 2012, Su et al. [19] introduced a sparse and redundant combination of algorithm for learned dictionary. It is used to process different types of images. They utilize the K-SVD denoising system and adjust its underlying lexicon, and after that for the most part center on utilizing it to think about its denoising execution and appropriateness for various sorts of Pictures, and after that contrast it and some other picture denoising calculations. With regards to the remote detecting pictures denoising, the analysis comes about demonstrate that the K-SVD calculation can prompts the condition of-craftsmanship denoising execution at low boisterous levels, yet for high loud levels, its execution isn't great on PSNR and visual impact, that is it can't hold the nearby points of interest of pictures.

In 2012, Zhang et al. [20] proposed an image denoising method which is based on support vector regression. The results show that the denoising is better than the previous method and it is capable in restoring the original image.

In 2017, Chithra and Santhanam [21] proposed a hybrid filter. It is used for suppressing the Gaussian noise in computer tomography (CT) for improving the quality of the image. Their method is the combination of modified median wiener filter (MMWF) and absolute difference and mean (ADMF) filter. It is compared with the triangular and direction-based filter (TDBF), discrete wavelet transform using total variation (DWTTV), edge preserving hybrid filter (EPHF) and ADMF filter. The result shows the proposed method is better in terms of peak signal to noise ratio (PSNR) and mean square error (MSE).

In 2017, Soni and Kirar [22] suggested the challenges found in noise removal. In the course of recent decades, various types of clamor lessening methods have been produced. They audits the change based denoising procedures and plays out their similar examination. They put consequences of various approaches including general ridgelets and curvelets, Experimental Mode Decomposition and Empirical ridgelets and curvelets. A quantitative measure of examinations is displayed as far as PSNR.

In 2017, Pang [23] suggested that the wavelet decomposing levels and the selection of the thresholding function may affect the performance of image denoising. They have presented a new approach for the wavelet decomposing levels identification using the 2D Haar wavelet thresholding method. It utilizes the standard deviation estimations of the sub-groups to see whether the flag vitality is solid or frail in the high recurrence sub-groups after the 2D Haar wavelet change. In expansion, another thresholding capacity is proposed which accomplishes better denoising execution as far as PSNR and MSE than the delicate thresholding technique. Particularly, at high commotion levels, the proposed new thresholding technique outflanks hard 
Shroff et al.

thresholding, delicate thresholding and semi-delicate thresholding techniques.

In 2017, Yang and Liu [24] suggested that the Fluorescence molecular imaging tomography (FMT) has the advantages of early intervention, low cost and easy use. FMT can obtain the appropriation territory of fluorophore in body without cutting the body or placing something into the body. However, fluorescence sub-atomic picture is extremely delicate to numerous sort of commotions, for example, autofluorescence, background noise a few different commotions.

The fluorescence flag is the real segment in fluorescence picture, the power of autofluorescence and other foundation signals are generally low in the analysis result. This technique dispense with high recurrence clamors by Gaussian smoothing in recurrence area, at that point, to extricate the significant segments of the fluorescence motions by wavelet change, at last, k-means grouping is used to isolate the significant part and foundation of the fluorescence picture. Trial comes about demonstrate that the proposed strategy is viability, it could get the fluorescence flag while wipe out the foundation clamor, and increment the nature of fluorescence picture.

In 2017, Ankarao et al. [25] suggested that the orthogonal matching algorithm is cost effective in terms of computational complexity. This calculation gives an answer for over determined also, underdetermined frameworks by limiting the blunder capacities utilizing slightest square. This work focuses on the development of lexicon which can be utilized to comprehend the sparsity based picture denoising issue.

They constructed the dictionary least square solution subjected to thresholding conditions orthogonal matching pursuit (OMP) calculation stays away from the determination of a similar particle in each emphasis, because of the presence of symmetrical property between the deposit and the iota chose from the word reference. Therefore, OMP calculation brings about exact picture reproduction.

The proposed strategy is approved on four standard test pictures, for example, Lena,Vessel, Barbara and Cameraman with various clamors, for example, salt and pepper commotion, Gaussian clamor and spot clamor with fluctuating the level of commotion level from $5 \%$ to $40 \%$. Gotten comes about are assessed by the quality metric PSNR and contrasted and the current wavelet based meager picture denoising.

The test assessment demonstrates that the proposed technique is better material to expel the dot commotion and salt and pepper clamor when contrasted and the current wavelet based inadequate picture denoising.

\section{Proposed approach}

In this paper a k-means based PDE (KPDE) has been applied for image denoising. In this approach first data pre-processing mechanism has been applied. In this process an image has been processed for preprocessing. The color balance of the image has been extracted and verified. Demosaicing algorithm has been used to reconstruct it with the complete color extraction. The incomplete pixel forms an overlaid with a color filter array. It fills the gap for the reconstruction. Then sharpening, color performance and transformation have been done. In this process the data formation in the fixed dimension for the further process has been formulated. Final data preprocessed values have been obtained. It is also shown in Figure 1.

The next procedure is for the image denoising. In this process the pre-processed image has been selected. Gaussian noise has been added in terms of percentage like $5 \%, 10 \%, 15 \%$ etc. Then k-means algorithm has been applied for the object based data clustering. Then block based decomposition has been performed based on the similarity ranking and matching.

Then the combination of PDE-FFT has been applied for the noise or in terms of error. It is separated based on the mean filter applied. It is separated based on the error and the mean filter applied. After reduction of the noise we then find the resultant image. PDE approach has the dependency on the neighboring approach and it can link through the adjacent point. So the removal of noise can be accurate if the proper adjacent neighbor position is identified. In our case due to $\mathrm{k}$-means it is easier.

Leena, Barbara and Wang database have been considered for the experimentation. The database of James Z. Wang [26] which is the collection of 1000 Databases has been used along with the other images. There are 10 categories in the database including African men, seas, Buildings, Buses, dinosaur, elephant, roses, horses, mountains and food. 


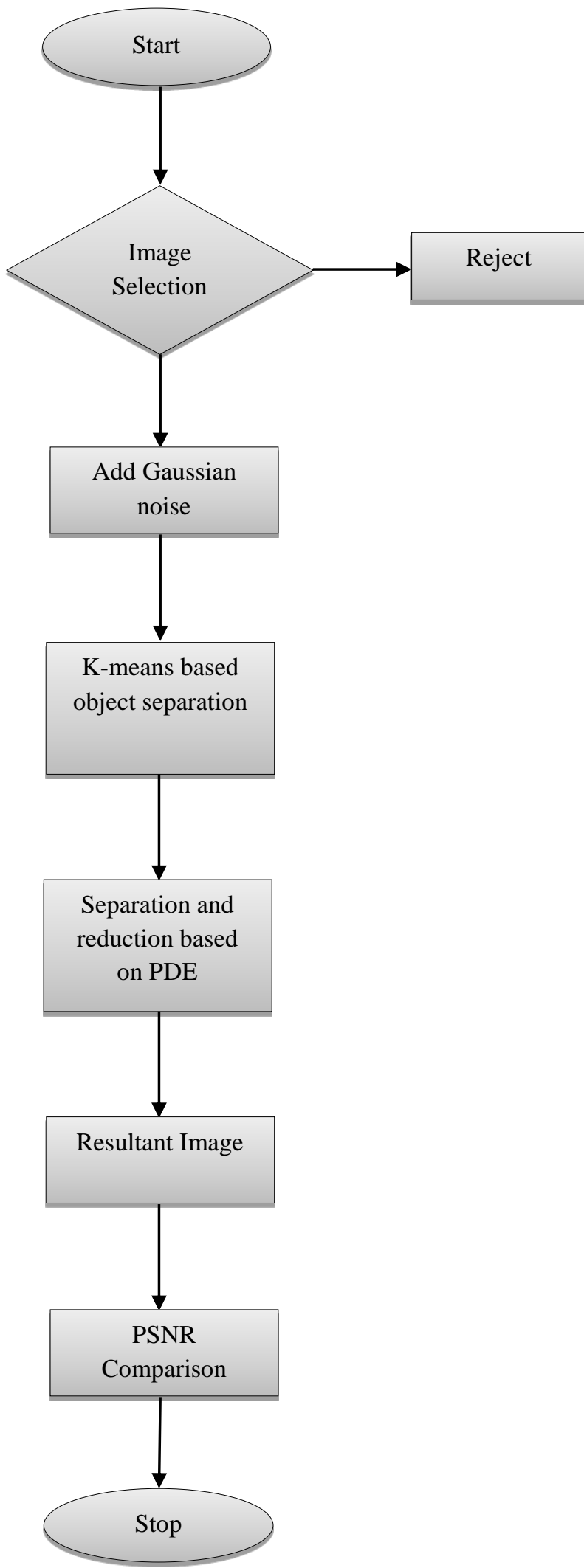

Algorithm of the proposed approach is shown below.

Input: Images of $256 \times 256$ and $512 \times 512$ with Gaussian noise

Output: Image retrieval after removing the Gaussian noise

Step 1: Input the image.

Step 2: Add white Gaussian noise.

Step 3: K-clusters have been determined first.

Step 4: Then centroid is selected.

Step 5: Euclidean distance is used for calculated based on the coordinates $(x, y)$ and $(a, b)$ is given by: $\operatorname{dist}((x, y),(a, b))=\sqrt{ }(x-a)^{2}+(y-b)^{2}$

Step 6: The remaining values are presently analyzed in succession and allotted to the bunch to which they are nearest.

Step 7: The information is selected out to the gathering that is neighboring the centroid.

Step 8: The centroids are recalculated till the set is not empty.

Step 9: PDE-FFT are then applied for the noise separation.

Step 10: Final image is constructed based on the noise separation.

Step 11: Image received as the output

Step 12: End

\section{Experimental results}

There are 10 categories in the database. We have considered several images along with the Wang database. We have used peak signal to noise ratio (PSNR) to evaluate the algorithm.

Mean square error $(\mathrm{MSE})=\frac{\sum_{M, N}\left[I_{1}(m, n)-I_{2}(m, n)\right]}{M \times N}$

$\mathrm{M}$ and $\mathrm{N}$ are the number of rows and columns

$\mathrm{PSNR}=10 \log _{10}\left(\frac{R^{2}}{M S E}\right)$

$\mathrm{R}$ is the maximum fluctuation in the input in the image data type.

We have compared the results from reference number [26]. The comparison is shown in Table 1 to 3 . It clearly shows that our approach has efficient capability of denoising from the traditional methods.

Table 1 PSNR values comparison in case of Leena (Noise 5\%)

\begin{tabular}{ll}
\hline Methods & PSNR \\
\hline$[25]$ & 20.7 \\
PDE & 21.7 \\
KPDE & 23.2 \\
KPDE + FFT & 23.7 \\
\hline
\end{tabular}

Figure 1Working process flowchart 
Shroff et al.

Table 2 PSNR values comparison in case of Barbara (Noise 5\%)

\begin{tabular}{ll}
\hline Methods & PSNR \\
\hline$[25]$ & 19.8 \\
PDE & 18.6 \\
KPDE & 20.6 \\
KPDE + FFT & 20.8 \\
\hline
\end{tabular}

Table 3 PSNR values comparison in case of Cameraman (Noise 5\%)

\begin{tabular}{ll}
\hline Methods & PSNR \\
\hline$[25]$ & 20.1 \\
PDE & 21.3 \\
KPDE & 23.4 \\
KPDE + FFT & 24.6 \\
\hline
\end{tabular}

\section{Conclusion}

$\mathrm{K}$-means based PDE has been proposed in this paper. It is used for the noise reduction. Data pre-processing has been applied and then it is arranged for the further process. For noise we have used Gaussian noise. It has been added in terms of noise percentage to find the efficiency in terms of PSNR values. The noise percentage has been added like 5\%,10\%, 15\% etc. Then k-means algorithm has been applied for the object based data clustering. Then block based decomposition has been performed based on the similarity ranking and matching. Then the combination of PDE-FFT has been applied for the noise or in terms of error. It is separated based on the error and the mean filter applied. After reduction of the noise we then find the resultant image. PDE approach has the dependency on the neighboring approach and it can link through the adjacent point. So the removal of noise can be accurate if the proper adjacent neighbor position is identified. In our case due to k-means it is easier. The results suggest that our approach has the capability of removing noise efficiently.

\section{Acknowledgment}

None.

\section{Conflicts of interest}

The authors have no conflicts of interest to declare.

\section{References}

[1] Shannon CE. Communication in the presence of noise. Proceedings of the IRE. 1949; 37(1):10-21.

[2] Nyquist H. Certain topics in telegraph transmission theory. Transactions of the American Institute of Electrical Engineers. 1928; 47(2):617-44.

[3] Candes EJ, Wakin MB. An introduction to compressive sampling. IEEE Signal Processing Magazine. 2008; 25(2):21-30.
[4] Ghosh P, Pandey A, Pati UC. Comparison of different feature detection techniques for image mosaicing. ACCENTS Transactions on Image Processing and Computer Vision. 2015; 1(1):1-7.

[5] Tropp JA, Gilbert AC. Signal recovery from random measurements via orthogonal matching pursuit. IEEE Transactions on Information Theory. 2007; 53(12):4655-66.

[6] Kumar M, Katti CP. An efficient ID-based partially blind signature scheme and application in electroniccash payment system. ACCENTS Transactions on Information Security. 2017; 2(6):36-42.

[7] Chitra AD, Ponmuthuramalingam P. Face recognition with positive and negative samples using support vector machine. ACCENTS Transactions on Image Processing and Computer Vision. 2016; 2(5):16-9.

[8] Mohapatra BN, Panda PP. Histogram equalization and noise removal process for enhancement of image. ACCENTS Transactions on Image Processing and Computer Vision. 2017; 3(9): 22-5.

[9] To AC, Moore JR, Glaser SD. Wavelet denoising techniques with applications to experimental geophysical data. Signal Processing. 2009; 89(2):14460.

[10] TV NP, Hemanth VK, Kumar S, Soman KP, Soman A. Comparative study of recent compressed sensing methodologies in astronomical images. In eco-friendly computing and communication systems 2012 (pp. 108-16). Springer, Berlin, Heidelberg.

[11] Dubey S, Hasan F, Shrivastava G. A hybrid method for image denoising based on wavelet thresholding and RBF network. International Journal of Advanced Computer Research. 2012; 2(4):167-72.

[12] Liua J, Shi C, Gao M. Image denoising based on BEMD and PDE. In international conference on computer research and development 2011 (pp. 110-2). IEEE.

[13] Motwani MC, Gadiya MC, Motwani RC, Harris FC. Survey of image denoising techniques. In proceedings of GSPX 2004 (pp. 27-30).

[14] Candes EJ, Tao T. Decoding by linear programming. IEEE Transactions on Information Theory. 2005; 51(12):4203-15.

[15] Singh J, Dubey RB. Reduction of noise image using LMMSE. International Journal of Advanced Computer Research. 2012; 2(5): 147-52.

[16] Anandan P, Sabeenian RS. Curvelet based image compression using support vector machine and core vector machine-a review. International Journal of Advanced Computer Research. 2014; 4(15):675-81.

[17] Veena PV, Devi GR, Sowmya V, Soman KP. Least square based image denoising using wavelet filters. Indian Journal of Science and Technology. 2016; 9(30):1-6.

[18] Lang C, Li G, Li J, Zhao X. Combined transform image denoising based on morphological component analysis. In international conference on multimedia technology 2011 (pp. 4871-4). IEEE.

[19] Su K, Fu H, Du B, Cheng H, Wang H, Zhang D. Image denoising based on learning over-complete 
dictionary. In international conference on fuzzy systems and knowledge discovery 2012 (pp. 395-8). IEEE.

[20] Zhang GD, Yang XH, Xu H, Lu DQ, Liu YX. Image denoising based on support vector machine. In spring congress on engineering and technology 2012 (pp. 14). IEEE.

[21] Chithra K, Santhanam T. Hybrid denoising technique for suppressing Gaussian noise in medical images. In IEEE international conference on power, control, signals and instrumentation engineering 2017 (pp. 1460-3). IEEE.

[22] Soni N, Kirar K. Transform based image denoising: a review. In international conference on recent innovations in signal processing and embedded systems 2017 (pp. 168-71). IEEE.

[23] Pang J. Improved image denoising based on Haar wavelet transform. In smartworld, ubiquitous intelligence \& computing, advanced \& trusted computed, scalable computing \& communications, cloud \& big data computing, internet of people and smart city innovation 2017. IEEE.
[24] Yang W, Liu J. Denoising fluorescence molecular image by $\mathrm{k}$-means clustering. In IEEE international conference on computer and communications 2017 (pp. 1847-50). IEEE.

[25] Ankarao V, Sowmya V, Soman KP. Sparse image denoising using dictionary constructed based on least square solution. In international conference on wireless communications, signal processing and networking 2017 (pp. 1165-71). IEEE.

[26] http://wang.ist.psu.edu/docs/related/ Access 23 March 2018.

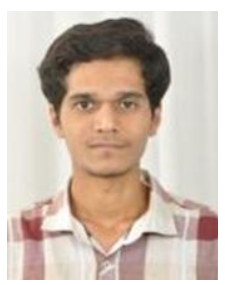

Mr. Abhishek Dipak Shroff completed B.E. in Computer Engineering from North Maharashtra University, Jalgaon in 2015 and he is currently pursuing M.tech in Computer Science and Engineering from Sri Satya Sai university of Technolgy and Medical Sciences, Sehore, MP, India.

Email: drabhishekshroff@gmail.com 